sind Verbindungen von Kohlenhydraten mit organischen Nichtkohlenhydraten, dis unter Aufnahme von Wasser in ihre Komponenten gespalten werden können. AlHaupteinteilungsprinzip wird die Konstitution der nicht kohlenhydratartigen Bestandteile ("Aglykone") benutzt; diese zerfallen in stickstofffreie, stickstoffhaltige sowie stick, stoff- und $\$ r$ wefelhaltige, die weiter gegliedert werden in aliphatische, hydrocyklische, aromatische und heterocyklische Verbindungen. Die Nebeneinteilung geschieht mit Hilfe der Kohlenhydrate. Eine Übersicht, enthaltend die bekanntesten, zumeist den Pflanzenglykosiden angehörenden Körper, läßt die Einzelheiten dieser Einteilungsart erkennen. G. Sonntag.

\title{
Honig.
}

C. A. Browne: Chemische Analyse und Zusammensetzung amerikanischer Honige. (Zeitschr. Ver. Deutsch. Zuckerind. 1908, 45, 751-806). - Verf. hat 100 Proben reinen Honigs aus 32 Staaten und Territorien, darunter 11 aus Hawaii, 4 aus New-England analysiert. Alle Proben waren vorher ausgeschleudert und durchgeseiht worden. Die Analysenergebnisse sind in Tabellen zusammengestellt, in Klassen nach ihrer Blütenabstammung, wie sie von den Imkern bezeichnet worden war, geordnet. Wenn diese Ordnung nur nominellen Wert besitzt, so zeigen doch die Zahlen einer jeden Honigklasse eine gewisse Übereinstimmung. In der nachstehenden Tabelle sind die Gesamtdurchschnitte und Grenzzahlen enthalten.

\begin{tabular}{|c|c|c|c|c|c|c|c|c|c|c|}
\hline \multicolumn{2}{|c|}{$\begin{array}{c}\text { Bezeichnung } \\
\text { der } \\
\text { Honige }\end{array}$} & 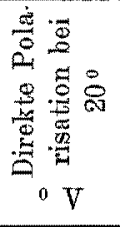 & 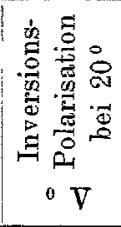 & 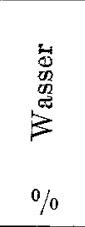 & 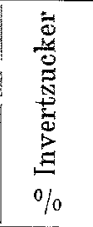 & 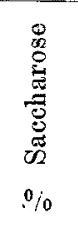 & $\begin{array}{l}\stackrel{g}{\tilde{y}} \\
\text { 品 } \\
\%\end{array}$ & 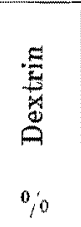 &  & 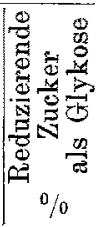 \\
\hline $\begin{array}{c}\text { Links- } \\
\text { drehende } \\
\text { Honige } \\
\text { (92 Proben) }\end{array}$ & $\begin{array}{c}\text { Höchst } \\
\text { Niedrigst } \\
\text { Mittel }\end{array}$ & $\begin{array}{l}-24,8 \\
-\quad 0,3 \\
-11,73\end{array}$ & $\begin{array}{r}-29,26 \\
-\quad 1,32 \\
-19,16\end{array}$ & $\begin{array}{l}26,88 \\
12,42 \\
17,70\end{array}$ & $\begin{array}{l}83,36 \\
62,23 \\
74,98\end{array}$ & $\begin{array}{c}10,01 \\
0 \\
1,90\end{array}$ & $\begin{array}{l}0,90 \\
0,08 \\
0,18\end{array}$ & $\begin{array}{l}7,58 \\
0,04 \\
1,51\end{array}$ & $\begin{array}{l}0,25 \\
0,04 \\
0,08\end{array}$ & $\begin{array}{l}79,86 \\
59,61 \\
71,08\end{array}$ \\
\hline $\begin{array}{l}\text { Rechts: } \\
\text { drehende } \\
\text { Honige } \\
\text { (7 Proben) }\end{array}$ & $\begin{array}{c}\text { Höchst } \\
\text { Niedrigst } \\
\text { Mittel }\end{array}$ & $\begin{array}{l}+17,75 \\
+\quad 3,6 \\
+\quad 9,43\end{array}$ & $\begin{array}{r}+14,96 \\
-\quad 2,53 \\
+\quad 5,47\end{array}$ & $\begin{array}{l}17,80 \\
13,56 \\
16,09\end{array}$ & $\begin{array}{l}71,69 \\
64,84 \\
66,96\end{array}$ & $\begin{array}{l}5,28 \\
0,61 \\
3,01\end{array}$ & $\begin{array}{l}1,29 \\
0,29 \\
0,81\end{array}$ & $\begin{array}{r}12,95 \\
6,02 \\
9,70\end{array}$ & $\begin{array}{l}0,19 \\
0,05 \\
0,12\end{array}$ & $\begin{array}{l}68,68 \\
62,12 \\
64,15\end{array}$ \\
\hline $\begin{array}{l}\text { Sümt } \\
(99 \mathrm{P}\end{array}$ & & $-13,02$ & $-17,41$ & 17,59 & 74,41 & 1,98 & 0,93 & 2,09 & 0,09 & 70,59 \\
\hline
\end{tabular}

Die angewandten Methoden werden näher beschrieben; die reduzierenden Zuckerarten wurden nach dem vom Verf. früher angegebenen Verfahren (Z. 1907, 13, 27) bestimmt, Dextrin in folgender Weise: $8 \mathrm{~g}$ Honig werden mit $4 \mathrm{ccm}$ Wasser in einen $100 \mathrm{ccm}$ Kolben gespült und mit absolutem Alkohol aufgefüllt. Wenn das Dextrin sich abgesetzt hat und die Flüssigkeit völlig klar geworden ist, wird sie durch ein Filter dekantiert, der Niederschlag mit $10 \mathrm{ccm} 95 \%$-igem Alkohol ausgewaschen, mit heißem Wasser gelöst, die Lösung eingedampft und der Rückstand im Wassertrockenschrank bis zum konstanten Gewicht getrocknet. Dann wird der Rückstand wieder mit Wasser zu einem bestimmten Volumen (das zebnfache des Gewichts vom Rückstand) gelöst und in aliquoten Teilen der filtrierten Lösung der Zucker vor und nach der Inversion bestimmt. Das Gewicht des Gesamtniederschlags vermindert um den Invertzucker und die Saccharose gibt den Gehalt an Dextrin. Diese Methode liefert 
genauere Werte, als die direkte Wägung der Alkoholfällung und ist besonders für vergleichende Untersuchungen von Wert, wenn sie auch nicht den wahren DextringehaIt angibt. - Aus der "Diskussion der Resultate" sei nachstehendes hervorgehoben: Die Farbe der Honige war vom reinsten Weib des Luzerne- und Kleehonigs bis zu der sehr dunklen Farbe des Buchweizenhonigs. 80\% der untersuchten Honige waren mehr oder weniger krystallisiert, mehr bei den Honigen von großer Reinheit, wie beim Luzernehonig, während der flüssige Zustand sich besonders bei den Honigtau-Honigen zeigte, und bei solchen von hohem Fructosegehalt (Salbeihonig). Geruch und Geschmack der verschiedenen Honige war gewöhnlich typisch für jede Klasse, in vielen Fällen war der Geruch der Blume, aus der der Nektar stammte, deutlich wahrnehmbar. Größere Mengen von Honigtau geben melassenartigen Geruch und Geschmack. - Sämtliche Honige zeigten Birotation, deren Größe (der Unterschied zwischen sofort abgelesener und der nach 18-20 Stunden beobachteten Polarisation) im Mittel $3,6^{\circ} \mathrm{V}$. betrug. - Höhere direkte Polarisation als $20^{\circ} \mathrm{V}$. ist kein Beweis für eine Verfälschung, denn 16 der untersuchten Honige polarisierten höher als $20^{\circ}$. - Mit Rücksicht auf zahlreiche mit der Verwendung der Clerge t'schen Formel bei der Berechnung des Zuckers im Honig verbundene Febler darf die Bestimmung nur nach dem gewichtsanalytischen Verfahren ausgeführt werden. - Die auch bei $97^{\circ}$ ausgeführten Polarisationen ergaben, daß die Differenz zwischen den Inversionspolarisationen bei $20^{\circ}$ und bei $87^{\circ}$ nahezu konstant für fast alle Honige ist: bei $80 \%$ der untersuchten Honige betrug sie $26-28^{\circ} \mathrm{V}$, bei $80^{\circ} \% 25-29^{\circ}$, bei fast 95\% 23-30, im Gesamtdurchschnitt war der Wert dieser Konstanten 26,710. Der Stickstoffgehalt von 7 verschiedenen Honigen betrug $0,017-0,090 \%$ Der Einflu der Umgebung auf die Zusammensetzung des Honigs zeigte sich darin, $\mathrm{da} B$ in Gegenden mit höherer Luftfeuchtigkeit und Regenmenge Honig von höherem Wassergehalt erzeugt wird. Die Hawaiischen Honige zeichnen sich durch einen hohen Gehalt an Chloriden aus und enthalten vielfach beträchtiche Mengen Honigtau. - In dem Abschnitt über Honigverfälschungen und deren $\mathrm{Nachwe}$ is teilt Verf. die Analyse eines Honigs mit, der bei Verfütterung von reiner Zuckerlösung gesammelt worden war; es waren ungefähr $80 \%$ des Zuckers invertiert. Wenn ein derartiger Honig mit Blütenhonig vermischt wird, so ist die Zuckerfütterung nicht nachzuweisen. Zum Nachweis von Stärkezucker empfiehlt Verf. die Beckmann'sche Probe auf Erythro- oder Amylodextrin mit Jod. Von den 100 analysierten Honigen gab keiner die Reaktion. Es wird aber darauf aufmerksam gemacht, daß es Kapillärsirupe und Maltosesirupe gibt. die die Reaktion mit Jod nicht zeigen. Bei der Besprechung der quantitativen Methoden kommt Verf. auf die Differenz in der Inversionspolarisation zwischen 20 und $87^{\circ}$ zurück. Diese Differenz ist bei reinem Honig ausschließlich eine Folge des Fructosegehaltes; der Zusatz eines Fructose nicht enthaltenden Fälschungsmittels würde ein Sinken der Polarisationsdifferenz, das der Menge dieses Mittels proportional ist, bewirken. Da die Differenz reiner Honige durch Schwankungen des Wassergehaltes, hohen Gehalt an Honigdextrinen und organischen Nichtzuckerstoffen erheblich beeinflubt wird, reduziert man die Polarisationsdifferenz auf reduzierenden Zucker und verfährt folgendermaßen: Die Inversionspolarisationsdifferenz zwischen 20 und $87^{\circ}$ wird mit 77 , dem Durchschnitts. gehalt reiner Honige an Invertzucker nach der Inversion, multipliziert und dieses Produkt dividiert durch den in der Probe gefundenen Invertzuckergehalt nach der" Inversion. Die so erhaltene korrigierte Polarisationsdifferenz gibt durch Multiplikation mit 100 und Division durch 26,7 den Gehalt an reinem Honig. Wie die an 5 Proben mit und ohne Zusatz von $20 \%$ Stärkezucker ausgeführten Untersuchungen zeigten, gibt auch diese Methode, wie zwei andere zum Vergleich herangezogene Berechnungsarten nur Näherungswerte. - Für den Nachweis von Invertzueker hat nach des Verf.'s Untersuchungen die Ley'sche Probe mit ammoniakalischer Silberlösung Wert als be- 
stätigende Probe, wenn man sich mit den bei reinen und künstlichen Honigen auftretenden Färbungen vertraut gemacht hat. Mit den 100 reinen Honigen gab das Reagens viel häufiger eine charakteristische, in der Intensität zwischen hellem Rot- und Purpurbraun schwankende Braunfärbung, während der grünliche Nachton bei sehr vielen Proben unbestimmt war. Als wertvolles Reagens erwies sich ferner die Anilin ace tatprobe: $5 \mathrm{ccm}$ Honiglösung 1:1 werden im Reagensglase mit 1 bis $2 \mathrm{ccm}$ Anilinacetatlösung ( $5 \mathrm{ccm}$ Anilin mit $5 \mathrm{ccm}$ Wasser und $2 \mathrm{ccm}$ Eisessig gelöst) überschichtet. Bildet sich ein roter Ring und verbreitet sich die rote Farbe allmählich durch die ganze Schicht der Anilinlösung, so ist künstlicher Invertzucker vorhanden. Die Probe fiel bei sämtlichen reinen Honigen negativ aus. Da die Reaktion auf dem bei der Herstellung des Invertzuckers durch die hohe Inversionstemperatur gebildeten Furfurol beruht, so reagieren auch alle Honige, die gekocht oder längere Zeit erhitzt wurden. Von den Ergebnissen seiner Untersuchungen einer großen Zahl von Honigen des Handels kann Verf, vorerst nur mitteilen, daß 20\% der Proben Glykose von geringen Mengen an bis zu $80 \%$ enthielten. 12\% der Proben überschritten einen Gehalt von $8 \%$ Sacebarose und $8 \%$ gaben die Reaktion auf künstlichen Invertzucker sowohl mit der Silberprobe als auch mit Anilinacetat. Büchsenhonige mit einer Blütenbezeichnung zeigen oft verdächtige Abweichungen gegenüber den Analysen derartiger Honige von bekannter Blütenherkunft; die mikroskopische Untersuchung ergab häufig völliges Fehlen der Pollen der fraglichen Blüte.

G. Somntag.

W. J. Young: Mikroskopische Untersuchung der Honigpollen. (Zeitschr. Ver. Deutsch. Zuckerind. 1908, 45, 806-820.) - Verf. hat den Pollen einer großen Anzahl von Blüten, von denen es bekannt oder anzunehmen war, daß sie von Bienen aufgesucht werden, aus verschiedenen Landesteilen Amerikas mikroskopisch untersucht. Da die Pollen von mehr oder weniger naho verwandten Pflanzen sehr ähulich sind, so sind die Pollen in verschiedene Typen, teils nach den Familien, teils nach Arten benannt, zusammengefalat und an der Hand von Mikrophotographien eingehend beschrieben. Die Ergebnisse dienten zum Vergleich mit den durch Zentrifugieren verdünnten Honigs erhaltenen Sedimenten. Zur Bestimmung der Zahl der in einem Honig vorhandenen Pollenkörner wird $1 \mathrm{~g}$ Honig in Wasser gelöst, zentrifugiert, die überstehende Flüssigkeit abgegossen und der Rest auf genau $1 \mathrm{ccm}$ aufgefüllt. 1 Tropfen der gut durchgemischten Flüssigkeit wird in eine Blutzählkammer gebracht und darin werden alle Pollenkörner gezählt. Es wurden 10 Zählungen vorgenommen, meist an zwei Proben und so bei 10 Honigproben Zahlen von 123 bis 5410 Pollenkörner in $1 \mathrm{~g}$ Honig gefunden. Es ergibt sich daraus, daß durch die Bestimmung der Anzahl der Pollenkörner ein Urteil über die Verfälschung eines Honigs nicht zu gegewinnen ist. In Honigsedimenten werden bisweilen nadelförmige Krystalle, Oktaeder und Prismen gefunden, die den im Harn gefundenen Calciumoxalat- und Phosphatkrystallen dem Aussehen nach gleich sind. Auch finden sich gewöhnlich Teile tierischer Herkunft: Haare, Schuppen u. a. von Insekten, auch Muskelfasern und Fragmente der Tracheen. Stets sind außer den Pollenkörnern andere Pflanzenteile vorhanden, Pilzsporen, Haare und andere Gewebsfragmente. Kleine Wabenteile finden sich häufig im Schleuderhonig, wenn der Honig genügend hoch erhitzt war, so sammelt sich das geschmolzene Wachs zu sphärischen Massen, die mit Pollenkörnern verwechselt werden können. Fast stets werden zufällige Verunreinigungen gefunden, wenn der Honig eine Zeitlang der Luft ausgesetzt war, worauf bei der Prüfung zur Aufdeckung von Verfälschungen Rücksicht zu nehmen ist. In mit Glykose verfälschten Honigen kommen oft Stärkekörner vor, die häufig durch Hitze verändert sind; diese können mit dem Fälschungsmittel hineingelangt sein.

G. Sonniag.

P. Soltsion: Zur Prüfung des Honigs. (Pharmaz. Ztg. 1907, 52, 1071.) - In Stärkesirup, Invertzucker und anderen Honigfälschungmitteln sind in der 
Regel Spuren von Eisen vorhanden, während dieses in reinem Honig gewöhnlich fehlt. Zur Prüfung auf diesen Bestandteil wird eine Lösung von 1 Teil Honig in 3 Teilen Wasser mit Essigsäure angesäuert und mit Ferrocyankaliumlösung versetzt. Reine Honige geben hierbei eine starke Trübung und bald einen weißlichen Niederschlag (Eiweifstoffe), die mit den genannten Surrogaten versetzten Honige eine schwächere Trübung, wobei der Niederschlag mehr oder weniger blau gefärbt ist. Es ist zu berüeksichtigen, daß auch reiner Honig durch Eisen z. B. in der Schleuder verunreinigt sein kann und daß andererseits weißer Rübenzuckersirup meist kein Eisen enthält.

A. Scholl.

Drawe: Beitrag zur Dr. Fiebe'schen Reaktion auf Invertzucker i m Hon i g. (Zeitschr. offentl. Chem. 1908, 14, 352.) - Ein selbstgeschleuderter Honig gab die Fiehe'sche Reaktion nicht; als er aber eine Stunde lang auf dem Wasserbade erbitzt war und nach dem Erkalten wiederum nach dem Fiehe'schen Verfahren geprüft wurde, trat starke Rotfärbung auf. Da im Honighandel die Erwärmung des Honigs nicht zu umgehen ist, ist die Reaktion nicht geeignet, eine Fälschung erkennen zu lassen. (Vergl. Z. 1908, 16, 517.)

G. Sonntag.

Utz: Verkauf von Zuckerfütterungshonig als Schleuderhonig is t nicht strafbar. (Zeitschr. offentl. Chem. 1908, 14, 171-174.) - Die vom Reichsgericht in vorstehendem Sinne gefällte Entscheidung muß schon deswegen befremden, weil sie sich in ausdrücklichen Widerspruch stellt mit der Definition, welche das Reichsgesundheitsamt dem Begriff Honig gibt, wonach dieser aus Blüten gesammelt sein soll. Auch der Schluh, der von den Bienen gern aufgenommene Zucker bleibe ein Naturprodukt, da er von ibnen zu Honig umgewandelt werde, ist nicht richtig, denn der Rohrzucker wird eben nicht zu Honig umgewandelt, sondern als solcher wieder abgeschieden. Der Hinweis auf den Instinkt der Bienen, welcher sie dazu verleite, den ihnen dargebotenen Robrzucker gern aufzunehmen, ist ebenfalls verfehlt, da hieraus nicht eine natürliche Veranlagung, sondern nur das Bestreben geschlossen werden kann, für das Geschäft des Honigsammelns möglichst wenig Arbeit zu verwenden, was allgemein der tierischen und menschlichen Natur entsprechen würde. Der Vergleich des Honigs mit der Kuhmilch ist ganz unzutreffend, da die Entstehung beider gänzlich verschieden ist. Verf. ist der Ansicht, daß dem Schwindel im Honighandel, welchem durch die Auslegungen des Reichsgerichts Tür und Tor geöffnet sej, nur dadurch entgegengetreten werden könne, daß das Publikum über diese Verhältnisse aufgeklärt und veranlaßt werde, nur "Blütenhonig“ $z$ fordern, sodaß der Zuckerfütterungshonig nur unter entsprechender Deklaration verkauft werden lürfe.

A. Seholl.

\section{Obst, Beerenfrüchte und Fruchtsäfte.}

J. M. Albahary: Neues Verfahren zur Trennung und Bestimmung der organischen Säuren in Früchten und Gemüsen. (Compt. rend. 1907, 144, 1232-1233.) - Die zu analysierenden Stoffe werden bei $100^{\circ}$ bis zum gleichbleibenden Gewicht getrocknet, fein gepulvert und im Soxhlet'schen Apparat nacheinander zuerst mit Chloroform, dann mit wasserfreiem Äther, zuletzt mit $90 \%$-igem Alkohol ausgezogen. Durch das Chloroform werden Fett, der größte Teil des Farb. stoffes und einige Alkaloide entfernt. In $100 \mathrm{~g}$ Äther sind löslich: $1,26 \mathrm{~g}$ Bernsteinsảure, 1,08 g Paraweinsäure, 9,1 g Citronensäure, $1,26 \mathrm{~g}$ Oxalsäure, 8,4 g Apfelsäure, Weinsäure in Spuren. $100 \mathrm{~g}$ Alkohol lösen 12,5 g Bernsteinsäure, 41,1 g Weinsäure, 20,8 g Paraweinsäure, 75,9 g Citronensäure, 12,81 g Oxalsäure, reichlich Äpfelsäure. Der ätherische und der alkoholische Auszug werden nach dem Abdampfen der Lösungsmittel vereinigt, in $100 \mathrm{ccm}$ Wasser gelöst und $10 \mathrm{ccm}$ dieser Lösung zur Bestimmung 